\title{
Pathogenic mechanisms of intracellular bacteria
}

\author{
Hans Helmut Niller ${ }^{\mathrm{a}}$, Roland Masa ${ }^{\mathrm{b}}$, Annamária Venkei \\ Sándor Mészáros ${ }^{\mathrm{b}}$, and Janos Minarovits ${ }^{\mathrm{b}}$
}

\begin{abstract}
Purpose of review
We wished to overview recent data on a subset of epigenetic changes elicited by intracellular bacteria in human cells. Reprogramming the gene expression pattern of various host cells may facilitate bacterial growth, survival, and spread.

Recent findings

DNA-(cytosine C5)-methyltransferases of Mycoplasma hyorhinis targeting cytosine-phosphate-guanine $(\mathrm{CpG}$ ) dinucleotides and a Mycobacterium tuberculosis methyltransferase targeting non-CpG sites methylated the host cell DNA and altered the pattern of gene expression. Gene silencing by CpG methylation and histone deacetylation, mediated by cellular enzymes, also occurred in M. tuberculosisinfected macrophages. M. tuberculosis elicited cell type-specific epigenetic changes: it caused increased DNA methylation in macrophages, but induced demethylation, deposition of euchromatic histone marks and activation of immune-related genes in dendritic cells. A secreted transposase of Acinetobacter baumannii silenced a cellular gene, whereas Mycobacterium leprae altered the epigenotype, phenotype, and fate of infected Schwann cells. The 'keystone pathogen' oral bacterium Porphyromonas gingivalis induced local DNA methylation and increased the level of histone acetylation in host cells. These epigenetic changes at the biofilm-gingiva interface may contribute to the development of periodontitis.
\end{abstract}

\section{Summary}

Epigenetic regulators produced by intracellular bacteria alter the epigenotype and gene expression pattern of host cells and play an important role in pathogenesis.

\section{Keywords}

bacterial effector, bacterial virulence, DNA methylation, epigenome, histone modification, pathoepigenetics

\section{INTRODUCTION}

Extracellular bacteria grow outside the cells of the infected host, whereas obligate intracellular bacteria enter into cells and use host cell resources for their replication [1]. Furthermore, a growing number of facultative intracellular bacteria can multiply both inside and outside host cells [2]. In addition to structural virulence factors, bacterial effector proteins that interact with the host may also facilitate disease initiation and progression [3,4,5"']. The transport of effectors from the bacterial cytosol into the host cells is typically mediated by translocation complexes called type III, type IV, and type VII secretion systems $\left[6^{\prime \prime}-9^{\prime \prime}, 10\right]$.

After entering the host cell, bacterial effector proteins may undergo covalent modification by cellular enzymes and their interaction with their targets may affect key processes of cellular physiology $\left[4,5^{-\prime}\right]$. Various bacterial effectors are located to discrete cellular compartments [5"']. A set of effector proteins is translocated to the nucleus and interacts with the chromatin $\left[11,12^{*}\right]$. Such effectors, some of them called nucleomodulins, may alter the cellular epigenotype and gene expression pattern $\left[13,14,15^{\mathbf{\prime}}, 16^{\mathbf{*}}, 17\right]$.

Although it is well documented that intracellular bacteria, including Campylobacter rectus, uropathogenic Escherichia coli, and Helicobacter pylori silence host genes by the induction of DNA hypermethylation, the exact mechanisms mediating epigenetic dysregulation by these pathogens has not been clarified $[18,19]$. Recent studies, however, elucidated the mechanism of de novo methylation in

anstitute for Medical Microbiology and Hygiene, University of Regensburg, Regensburg, Germany and 'bepartment of Oral Biology and Experimental Dental Research, Faculty of Dentistry, University of Szeged, Szeged, Hungary

Correspondence to Janos Minarovits, MD, PhD, Department of Oral Biology and Experimental Dental Research, Faculty of Dentistry, University of Szeged, Szeged, Hungary. Tel: +36 703948 279;

e-mail: minimicrobi@hotmail.com

Curr Opin Infect Dis 2017, 30:000-000

DOI:10.1097/QCO.0000000000000363 


\section{KEY POINTS}

- Bacterial DNMTs target the host cell DNA and alter the host cell methylome.

- Intracellular bacteria may possess an array of epigenetic regulators that target the cellular epigenetic machinery.

- The epigenetic alterations elicited by the same bacterial pathogen depend on the host cell phenotype.

- Epigenetic regulators produced by intracellular bacteria may facilitate bacterial growth, survival, and spread.

- The epigenetic changes induced by intracellular bacteria may serve as diagnostic tools and as targets of epigenetic therapy.

cells infected by Mycoplasma hyorhinis and Mycobacterium tuberculosis $\left[20^{\mathbf{\prime}}, 21,22,23^{-"}\right]$.

\section{Epigenetic reprogramming of host cells by Mycoplasma hyorhinis DNA methyltransferases}

Although Mycoplasma was considered as extracellular bacteria, their reduced genome may reflect a strictly parasitic lifestyle [24"]. Mycoplasma species either adhere to host cells or invade cells and replicate intracellularly for prolonged periods $\left[25^{\circ}, 26^{\prime \prime}\right]$. Mycoplasma salivarium was detected in the epithelial cells of oral leukoplakia, a premalignant condition of the oral cavity, whereas Mycoplasma hominis infection was associated with prostate cancer $\left[27,28^{*}\right]$.

Several Mycoplasma species including M. hyorhinis, encode DNA-(cytosine-5)-methyltransferase (DNMT) enzymes that target cytosine-phosphate-guanine (CpG) dinucleotides, similarly to their mammalian counterparts $\left[20^{\mathbf{\prime}}, 29,30\right]$. These enzymes establish the methylation patterns of the bacterial genomes. When expressed in human cells, they are also capable to translocate into the cell nucleus and create aberrant methylation patterns of the host cell DNA $\left[20^{-\bar{*}}, 21\right]$.

Chernov et al. [21] demonstrated that expression of Mhy1, a M. hyorhinis gene coding for a CpG-specific bacterial DNMT caused genome-wide methylation changes of the host DNA in transfected human fibrosarcoma and trophoblast cells. In parallel, the expression of certain cellular genes was upregulated, whereas another gene set showed a decreased level of transcription. All of these changes resulted in the activation of proliferation-specific pathways. A dual-specific DNMT encoded by Mhy2 was also active in human cells, it methylated both CpG dinucleotides and cytosines in a non-CpG sequence [21].
A subset of CpG dinucleotides was also modified in human cells by Mhy3, a M. hyorhinis methylase targeting GATC sequences [21]. 'Reprogramming' of the host epigenome by Mycoplasma species may contribute to the initiation or progression of malignant tumours [21,31",32].

\section{Alteration of the host cell epigenotype by Mycobacterium tuberculosis: noncanonical and classical epigenetic mechanisms}

M. tuberculosis, the causative agent of tuberculosis, infects humans by the respiratory route. Although the bacterium is engulfed by alveolar macrophages, its survival strategies, such as the inhibition of phagosome-lysosome fusion, allow intracellular persistence and replication [33,34",35"]. Sharma et al. [22] observed that Rv2966c, a secreted protein encoded by the genome of $M$. tuberculosis could enter human monocytic cell line derived from an acute monocytic leukemia patient (THP1) cells. Following nuclear transport, Rv2966c methylated the host cell DNA at cytosines located outside CpG dinucleotides, typically at cytosine-phosphate-adenine and cytosinephosphate-thymine sites [22]. Methylation at nonCpG sites occurs in stem cells and neoplastic cells in mammals, but it is usually absent from adult differentiated cell types, with few exceptions [36].

Sharma et al. [22] argued that non-CpG methylation could persist for a prolonged period in the genome of $M$. tuberculosis infected, nondividing macrophages. Non-CpG methylation in the intronic region of the histone variant gene $H 2 A F Y 2$ and in the GRK5 gene encoding a $\mathrm{G}$ protein-coupled receptor kinase caused transcriptional repression. Rv2966c could bind to specific DNA sequences and interacted with the activating chromatin marks H3K4me3 and H3K36me3 (histone H3 trimethylated at lysine 4 and 36, respectively) [22]. In a follow-up study, Sharma et al. [23"'] observed genome-wide de novo methylation at non-CpG dinucleotides in THP1 macrophages infected with M. tuberculosis strain H37Rv. In addition to hypermethylated regions, hypomethylated regions were also detected. Differentially methylated regions, especially hypermethylated sequences, frequently carried a 28 base pair motif with a conserved 'GCCTCC' core sequence [23"']. The H37Rv-induced changes in the host cell epigenome and transcriptome may establish a favourable environment for the intracellular persistence of the bacterium.

Infection of THP-1-derived macrophages with clinical isolates of M. tuberculosis-induced hypermethylation at $\mathrm{CpG}$ dinucleotides in promoter sequences of several interleukin receptor genes, including the promoter for interleukin 6 receptor [37"]. This 
epigenetic change may downregulate the level of interleukin 6 receptor protein mediating the effects of the pleiotropic regulator interleukin 6 .

In addition to an unusual non-CpG DNA methylase, the genome of $M$. tuberculosis encodes an 'unorthodox' protein arginine methyltransferase, too. Unlike its eukaryotic counterparts that target the long N-terminal tail of histones, the bacterial enzyme Rv1988 dimethylated an arginine residue located to the core region of histone H3 [38]. Rv1988 repressed a series of host defence genes coding for enzymes generating reactive oxygen species and nitric oxide. The expression of tumour necrosis factor receptor-associated factor 3, coding for an ubiquitin ligase of the tumour necrosis factor receptor-associated factor family, was also suppressed [38]. Thus, a bacterial histone arginine methyltransferase may block host defences, possibly promoting thereby pathogen survival.

A classical epigenetic mechanism, promoter silencing by the upregulation of the cellular histone deacetylase 1, was also observed in M. tuberculosisinfected macrophages [39]. Silencing of the interleukin- $12 \beta$ promoter was accompanied by the recruitment of HDAC1 and histone $\mathrm{H} 3$ deacetylation. Thus, M. tuberculosis may suppress the expression of a key regulator of the T helper 1 response by epigenetic modification [39].

M. tuberculosis may also elicit, however, histone acetylation, because Rv3423.1, a bacterial histone acetyltransferase could be detected in the chromatin of infected macrophages [40"]. Rv3423.1 generated euchromatic histone marks by acetylating histone $\mathrm{H} 3$ at lysine K9 and K14.

In contrast to THP-1-derived macrophages, human monocyte-derived dendritic cells permit only a constrained intracellular survival for $M$. tuberculosis [41]. The cellular epigenome in M. tuberculosis-infected dendritic cells was characterized by demethylation at distal enhancer elements and an increased level of activating histone modifications [42]. Transcriptional activation of the immune-related genes REL, CD83, and NFKB1 occurred before DNA demethylation [42]. Thus, the epigenetic alterations induced by $M$. tuberculosis are cell type specific, that is, they differ in macrophages and dendritic cells (Table 1).

Identification of epigenetic 'signatures' in granulocytes and monocytes of patients with active tuberculosis and latent $M$. tuberculosis infection may help to monitor the development and progression of tuberculosis [43]. Because epigenetic processes are reversible, microbe-induced epigenetic alterations are potential targets of epigenetic therapy.

\section{Induction of promoter hypermethylation in host cells by an Acinetobacter baumannii transposase}

The genome of Acinetobacter baumannii, a nonoral facultative intracellular bacterium involved in nosocomial infections and periodontitis, encodes a transposase protein that is associated with secreted outer membrane vesicles (OMVs) $[44,45]$. After delivery via OMVs to host cells, the transposase translocates to the nucleus and acts as a bacterial effector by inducing hypermethylation and silencing of the cellular E-cadherin promoter [45]. Thus, A. baumannii may induce epigenetic alterations and downregulation of the tumour suppressor protein Ecadherin in uninfected host cells that take up OMVs. Further studies may reveal the exact mechanism of transposase-mediated CpG methylation.

\section{Epigenetic reprogramming of host cells by Mycobacterium leprae, an obligate intracellular bacterium}

Intracellular bacteria including Helicobacter pylori, Lactobacillus acidophilus, and Mycobacterium leprae may alter the phenotype and fate of somatic cells

Table 1. Mycobacterium tuberculosis induced epigenetic alterations in host cells: recent advances

\begin{tabular}{llll}
\hline Effector & Function & Effect & Reference \\
\hline Rv2966c & $\begin{array}{c}\text { DNA-(cytosine-5)-methyltransferase targeting CpA } \\
\text { and CpT }\end{array}$ & Gene silencing in macrophages & [22,23"'] \\
ND & $\begin{array}{l}\text { Induction of DNA methylation at CpG dinucleotides } \\
\text { Rv1988 }\end{array}$ & $\begin{array}{l}\text { Protein arginine methyltransferase dimethylating } \\
\text { histone H3 arginine 42 (H3R42) }\end{array}$ & Gene silencing (?) in macrophages \\
ND & $\begin{array}{l}\text { Upregulation of HDAC1 (cellular histone } \\
\text { deacetylase) }\end{array}$ & Gene silencing in macrophages \\
Rv3423.1 & $\begin{array}{l}\text { Bacterial histone acetyltransferase } \\
\text { Induction of DNA demethylation and deposition of } \\
\text { euchromatic histone marks }\end{array}$ & Gene activation (?) in macrophages \\
\hline
\end{tabular}

CpA, cytosine-phosphate-adenine; CpG, cytosine-phosphate-guanine; CpT, cytosine-phosphate-thymine; HDACl, histone deacetylase; ND, not determined. 
[46]. In an experimental model of human leprosy, $M$. leprae infection of mouse primary Schwann cells caused downregulation of the master regulator Sryrelated HMG (high mobility group) box 10 in host cell nuclei, possibly because of bacterium-induced, methylation-mediated silencing of the Sox10 gene [47]. In parallel, the transcription of the Sox10-regulated myelin genes decreased, whereas a series of genes involved in epithelial-mesenchymal transition (EMT) were demethylated and activated. Thus, the M. leprae-infected Schwann cells acquired the phenotype of progenitor/stem-like cells. Accordingly, they could differentiate into myofibers and smooth muscles both in vitro and in vivo, a phenomenon that may facilitate the dissemination of bacteria $[47,48]$.

The exact mechanism of $M$. leprae-induced reprogramming remains to be elucidated. We suggest that EMT was initiated by the virulence factor phenolic glycolipid (PGL) which is loosely linked to the outer cell wall layer of the bacterium. PGL can modulate macrophage function by reducing the levels of inflammatory mediators (tumor necrosis factor- $\alpha$, interleukin 6 , and interleukin$1 \beta)$ released from the activated cells [49"]. Other candidates for the induction of EMT are the putative ligands binding to Toll-like receptor 4 (TLR4) [50"]. These molecules comprise lipooligosacharides, proline-glutamate/proline-proline-glutamate

proteins that are abundant in pathogenic mycobacteria, and putative M. leprae orthologs of TLR4-binding proteins characterized in M. tuberculosis [50"]. The function of a number of proteins encoded by the $M$. leprae genome is unknown at present [51]. Their characterization may reveal further important aspects of M. leprae-host cell interactions.

\section{Epigenetic alterations induced by oral bacteria}

Polymicrobial communities of oral bacteria regularly form biofilms on surfaces within the mouth [52"']. Dental plaques, the biofilms located to the tooth surface play a crucial role in the development of such highly prevalent oral diseases as caries, gingivitis, and periodontitis [53]. Oral bacteria were implicated in the pathogenesis of oral carcinoma, too $\left[5^{\prime \prime}, 5^{\prime}\right]$. Moreover, it was suggested that Porphyromonas gingivalis, the 'keystone pathogen' of periodontitis and Fusobacterium nucleatum promote colorectal carcinogenesis [56"].

$P$. gingivalis, a low-abundance biofilm species is capable to trigger an inflammatory response and impair, in parallel, various host defence mechanisms $\left[53,57^{\mathbf{*}}, 5^{*}\right]$. These changes allow enhanced replication of other bacteria located to the dental biofilm. The relative abundance of individual members of the bacterial community is also altered, resulting in periodontitis, a dysbiotic disease. Periodontitis is characterized by complement activation, tissue destruction, increased efflux of gingival crevicular fluid, periodontal pocket formation, and alveolar bone loss [59,60"]. P. gingivalis excerts a disproportionately large effect on the structure of the microbial community resulting in the transition of a 'healthy' plaque into a 'pathological' plaque $[53,59]$. This keystone pathogen coaggregates with other bacteria and alters the gene expression pattern of the microbial community $\left[61,62,63^{\prime}, 64^{\prime \prime}\right.$.

In addition to interacting with bacteria located to the dental plaque, $P$. gingivalis also adheres to gingival epithelial cells (GECs) and persists and multiplies intracellularly $\left[65,66^{\prime}\right]$. After internalization into early phagosomes the bacterium is protected from immune mechanisms, and by the activation of cellular autophagy, it gets access to host proteins that are utilized as sources of carbon and nitrogen. P. gingivalis is able to exit the host cell and reenter into neighbouring cells [66"]. A recent study demonstrated that a prolonged interaction with $P$. gingivalis increased the methylation of the TLR2 promoter in GECs [67]. Using a reporter construct, the activity of the methylated TLR2 promoter was reduced compared with the unmethylated one. Because TLR2 is involved in the recognition of the pathogen and it is indispensable for the upregulation of cytokine and antimicrobial peptide production following bacterial stimulation, methylation-mediated inhibition of TLR2 expression may result in innate immune dysbiosis manifested as hyporesponsibility of the GECs to $P$. gingivalis [67].

Short-term interaction of lipopolysacharide (LPS) derived from $P$. gingivalis with human oral keratinocytes also induced epigenetic alterations. Martins et al. [68] found that the level of acetylated histone $\mathrm{H} 3$, a euchromatic mark associated with active promoters, increased quickly following LPS exposure.

Coinfection by P. gingivalis and Filifactor alocis, a Gram-positive anaerobic rod associated with periodontitis, downregulated the level of histone $\mathrm{H} 1$ family members in epithelial cells $[69,70]$. This may alter the structure of nucleosome and the host cell transcriptome.

In addition to $P$. gingivalis-derived LPS, shortchain fatty acids (SCFAs) secreted by the bacterium may also increase histone acetylation and activate promoters in host cells. Furthermore, SCFAs inhibit histone deacetylase enzymes that remove acetyl moieties from histone tails, and suppress histone N-lysine methyltransferases that create heterochromatic marks at inactive promoters. SCFAs produced by $P$. gingivalis and Fusobacterium nucleatum reactivated latent, epigenetically modified herpesvirus genomes: 
they switched on the lytic (productive) replication cycle of Kaposi's sarcoma-associated herpesvirus and Epstein-Barr virus (EBV) [71,72]. In HIV- infected individuals activation of Kaposi's sarcoma-associated herpesvirus replication may contribute to the development of oral Kaposi's sarcoma, whereas EBV infection was associated with chronic periodontitis and with the presence of $P$. gingivalis in Japanese patients [73]. In pregnant women, coinfection of EBV and $P$. gingivalis may also facilitate the development of chronic periodontitis [74].

Thus, bacterium-host cell interactions may contribute to the epigenetic reprogramming of host cells at the biofilm-gingiva interface [71]. The altered host cell epigenotype and gene expression pattern as well as viral coinfections amplified by epigenetic regulators of bacterial origin may play a role in the progression of periodontitis $[71,72]$.

\section{Mobilization of chromosomally integrated human herpesvirus 6 genome by Chlamydia trachomatis}

The termini of the linear viral genomes of human herpesvirus (HHV) 6 and HHV7 consist of repetitive sequences, similarly to the human telomeric hexanucleotide TTAGGG. Both HHV6 and, HHV7 can integrate to subtelomeric chromosomal regions and have the ability to enter the human germline [75"]. About $0.5 \%$ of the population carries silent, chromosomally integrated HHV6 genomes that are transmitted vertically in a Mendelian fashion. Under specific conditions, such as pregnancy or immune suppression, chromosomally integrated HHV6 genomes may be reactivated and become infectious again, causing disease [76,77].

Chlamydia trachomatis, an obligate intracellular Gram-negative bacterium, causes inflammatory diseases. It uses the ephrin receptor tyrosine kinase A2 as a receptor for cellular entry [78]. Strangely enough, this receptor is also used by hepatitis $\mathrm{C}$ virus and HHV8 $[79,80]$. A nuclear effector protein secreted by $C$. trachomatis has histone methyl-transferase activity targeting histones $\mathrm{H} 2 \mathrm{~B}, \mathrm{H} 3$, and $\mathrm{H} 4$ (reviewed in [81]). Because $C$. trachomatis is sexually transmitted and is able to interfere with DNA damage response and telomeric damage response signalling, it was implicated in ovarian carcinogenesis [82]. In vitro, C. trachomatis superinfection is able to mobilize silenced chromosomally integrated HHV6 genomes from their subtelomeric locations via telomeric circle formation [83]. The unexpected interplay between $C$. trachomatis and HHV6 may be of great importance and warrants further experiments.

\section{CONCLUSION}

A diverse set of intracellular bacteria causes epigenetic dysregulation in host cells that contributes to disease initiation and progression.

\section{Acknowledgements}

We thank Professor Dr. Kornel Kovacs (University of Szeged, Faculty of Dentistry, Szeged, Hungary) for critical reading of the manuscript.

\section{Financial support and sponsorship}

The work was supported by the grant GINOP-2.3.2-152016-00011 to a consortium led by the University of Szeged, Szeged, Hungary (participants: the University of Debrecen, Debrecen, and the Biological Research Centre, Hungarian Academy of Sciences, Szeged, Hungary), project leader J.M. The grant was funded by the European Regional Development Fund of the European Union and managed in the framework of Economic Development and Innovation Operational Programme by the Ministry of National Economy, National Research, Development and Innovation Office, Budapest, Hungary.

\section{Conflicts of interest}

There are no conflicts of interest.

\section{REFERENCES AND RECOMMENDED \\ READING}

Papers of particular interest, published within the annual period of review, have been highlighted as:

- of special interest

- of outstanding interest

1. Silva MT. Classical labeling of bacterial pathogens according to their lifestyle in the host: inconsistencies and alternatives. Front Microbiol 2012; 3:71.

2. Ribet $D$, Cossart $P$. How bacterial pathogens colonize their hosts and invade deeper tissues. Microbes Infect 2015; 17:173-183.

3. Botta GA, Arzese A, Minisini R, Trani G. Role of structural and extracellular virulence factors in gram-negative anaerobic bacteria. Clin Infect Dis 1994; 18 (Suppl 4):S260-S264.

4. Asrat $\mathrm{S}$, Davis $\mathrm{KM}$, Isberg RR. Modulation of the host innate immune and inflammatory response by translocated bacterial proteins. Cell Microbiol $2015 ; 17: 785-795$.

5. Popa CM, Tabuchi M, Valls M. Modification of bacterial effector proteins - inside eukaryotic host cells. Front Cell Infect Microbiol 2016; 6:73.

The study reviews how interaction with host factors may activate the enzymatic activity of translocated bacterial proteins and describes how covalently modified bacterial effectors are targeted to various cellular compartments.

6. Dietsche T, Tesfazgi Mebrhatu M, Brunner MJ, et al. Structural and functional

- characterization of the bacterial type III secretion export apparatus. PLoS Pathog 2016; 12:e1006071.

The study describes the interactions and placement of proteins within the type III protein secretion system of Salmonella Typhimurium and presents a model for the assembly of the export apparatus.

7. Gaytan MO, Martinez-Santos VI, Soto E, González-Pedrajo B. Type three

- secretion system in attaching and effacing pathogens. Front Cell Infect Microbiol 2016; 6:129.

The review synthesizes available data regarding the assembly of the injectisome and secretion regulation in human and murine diarrheagenic bacteria.

8. Gonzalez-Rivera C, Bhatty M, Christie PJ. Mechanism and function of type IV - secretion during infection of the human host. Microbiol Spectr 2016; 4:.

The review covers the major subfamilies of type IV secretion systems including the effector translocator systems of intracellular Gram-negative bacteria.

9. Shah S, Briken V. Modular organization of the ESX-5 secretion system in - Mycobacterium tuberculosis. Front Cell Infect Microbiol 2016; 6:49.

The article compares the various type VII secretion systems of $M$. tuberculosis that are involved in the export of virulence proteins. 
10. Zhang XL, Li DF, Fleming J, et al. Core component EccB1 of the Mycobacterium tuberculosis type VII secretion system is a periplasmic ATPase. FASEB J 2015; 29:4804-4814.

11. Rennoll-Bankert KE, Garcia-Garcia JC, Sinclair SH, Dumler JS. Chromatinbound bacterial effector ankyrin A recruits histone deacetylase 1 and modifies host gene expression. Cell Microbiol 2015; 17:1640-1652.

12. Niller $\mathrm{HH}$, Minarovits J. Patho-epigenetics of infectious diseases caused by - intracellular bacteria. Adv Exp Med Biol 2016; 879:107-130.

The review covers major observations, till 2015, on epigenetic alterations induced by bacterial pathogens in host cells.

13. Li T, Lu Q, Wang G, et al. SET-domain bacterial effectors target heterochromatin protein 1 to activate host rDNA transcription. EMBO Rep 2013; 14:733-740.

14. Sinclair SH, Yegnasubramanian S, Dumler JS. Global DNA methylation changes and differential gene expression in Anaplasma phagocytophiluminfected human neutrophils. Clin Epigenetics 2015; 7:77.

15. Dumler JS, Sinclair SH, Pappas-Brown V, Shetty AC. Genome-wide Ana-

- plasma phagocytophilum AnkA-DNA interactions are enriched in intergenic regions and gene promoters and correlate with infection-induced differential gene expression. Front Cell Infect Microbiol 2016; 6:97.

The study describes in vitro binding of a rickettsial effector protein to the genomic DNA of human peripheral blood mononuclear cells and demonstrates its interac-

tions with putative matrix attachment regions and lamina-associated domains.

16. Farris TR, Dunphy PS, Zhu B, et al. Ehrlichia chaffeensis TRP32 is a

- nucleomodulin that directly regulates expression of host genes governing differentiation and proliferation. Infect Immun 2016.

The study describes nuclear transport, DNA binding and target genes of an effector protein encoded by the genome of Ehrlichia chaffeensis.

17. Sinclair SH, Garcia-Garcia JC, Dumler JS. Bioinformatic and mass spectrometry identification of Anaplasma phagocytophilum proteins translocated into host cell nuclei. Front Microbiol 2015; 6:55.

18. Minarovits J. Microbe-induced epigenetic alterations in host cells: the coming era of patho-epigenetics of microbial infections. A review. Acta Microbiol Immunol Hung 2009; 56:1-19.

19. Niller $\mathrm{HH}$, Banati F, Ay E, Minarovits J. Microbe-induced epigenetic alterations. In: Minarovits J, Niller HH, editors. Patho-epigenetics of disease. New York: Springer; 2012. pp. 419-455.

20. Chernov AV, Reyes L, Peterson S, Strongin AY. Depletion of CG-specific

I. methylation in Mycoplasma hyorhinis genomic DNA after host cell invasion. PLoS One 2015; 10:e0142529.

The study described the methylation pattern of the $M$. hyorhinis genome in free living bacteria and in bacteria internalized by human cells. The methylation pattern of the infected host cells was also determined. It was observed that the bacterial CG and GATC-specific DNMTs changed the host cell methylome.

21. Chernov AV, Reyes $L, X u Z$, et al. Mycoplasma CG- and GATC-specific DNA methyltransferases selectively and efficiently methylate the host genome and alter the epigenetic landscape in human cells. Epigenetics 2015; 10:303-318.

22. Sharma G, Upadhyay $S$, Srilalitha $M$, et al. The interaction of mycobacterial protein Rv2966c with host chromatin is mediated through non-CpG methylation and histone $\mathrm{H} 3 / \mathrm{H} 4$ binding. Nucleic Acids Res 2015; 43: 3922-3937.

23. Sharma G, Sowpati DT, Singh P, et al. Genome-wide non-CpG methylation of

-1. the host genome during M. tuberculosis infection. Sci Rep 2016; 6:25006.

The study describes how the host cell methyloma of THP1 macrophages changed after in vitro $M$. tuberculosis infection. Differential methylation at non-CpG dinucleotides was observed in immune response genes and genes involved in the reorganization of chromatin.

24. Chen $\mathrm{WH}$, van Noort $\mathrm{V}$, Lluch-Senar $\mathrm{M}$, et al. Integration of multi-omics data of

- a genome-reduced bacterium: prevalence of post-transcriptional regulation and its correlation with protein abundances. Nucleic Acids Res 2016; 44:1192-1202.

The study analysed a large number of data sets to reveal correlates of genome reduction in bacteria.

25. Martinelli L, Garcia-Morales L, Querol E, et al. Structure-guided mutations in

the terminal organelle protein MG491 cause major motility and morphologic alterations on mycoplasma genitalium. PLoS Pathog 2016; 12:e1005533.

The study presents the crystal structure of a Mycoplasma protein, a component of the terminal organelle involved in cell adhesion.

26. Shimizu T. Inflammation-inducing factors of Mycoplasma pneumoniae. Front - Microbiol 2016; 7:414.

The article reviews, among other mechanisms, how internalized Mycoplasma pneumoniae induces inflammation.

27. Mizuki H, Kawamura T, Nagasawa D. In situ immunohistochemical detection of intracellular Mycoplasma salivarium in the epithelial cells of oral leukoplakia. J Oral Pathol Med 2015; 44:134-144.

28. Khan $\mathrm{S}$, Zakariah $\mathrm{M}$, Rolfo $\mathrm{C}$, et al. Prediction of mycoplasma hominis proteins

- targeting in mitochondria and cytoplasm of host cells and their implication in prostate cancer etiology. Oncotarget 2016.

The study lists mycoplasma-encoded DNA-binding proteins and gene expression regulators implicated in tumorigenesis.

29. Luo W, Tu AH, Cao Z, et al. Identification of an isoschizomer of the Hhal DNA methyltransferase in Mycoplasma arthritidis. FEMS Microbiol Lett 2009; 290:195-198.
30. Wojciechowski M, Czapinska H, Bochtler M. CpG underrepresentation and the bacterial CpG-specific DNA methyltransferase M.Mpel. Proc Natl Acad Sci U S A 2013; 110:105-110.

31. Gomersall AC, Phan HA, lacuone $S$, et al. The Mycoplasma hyorhinis p37

- protein rapidly induces genes in fibroblasts associated with inflammation and cancer. PLoS One 2015; 10:e0140753.

The study describes a Mycoplasma surface protein involved in the induction of inflammatory proteins implicated in tumorigenesis.

32. Vande Voorde J, Vervaeke $P$, Liekens S, Balzarini J. Mycoplasma hyorhinisencoded cytidine deaminase efficiently inactivates cytosine-based anticancer drugs. FEBS Open Bio 2015; 5:634-639.

33. Fineran $P$, Lloyd-Evans $E$, Lack NA, et al. Pathogenic mycobacteria achieve cellular persistence by inhibiting the Niemann-Pick type $C$ disease cellular pathway. Wellcome Open Res 2016; 1:18

34. Awuh JA, Flo TH. Molecular basis of mycobacterial survival in macrophages.

- Cell Mol Life Sci 2016

The review deals with intracellular survival strategies of Mycobacteria.

35. Pires D, Marques J, Pombo JP, et al. Role of cathepsins in Mycobacterium

- tuberculosis survival in human macrophages. Sci Rep 2016; 6:32247.

The study deals with the role of cathepsins in pathogen killing.

36. Pinney SE. Mammalian non-CpG methylation: stem cells and beyond. Biology (Basel) 2014; 3:739-751.

37. Zheng L, Leung ET, Wong HK, et al. Unraveling methylation changes of host

- macrophages in Mycobacterium tuberculosis infection. Tuberculosis (Edinb) 2016; 98:139-148.

The study analysed methylation changes in in-vitro infected macrophages and macrophages from M. tuberculosis-infected patients.

38. Yaseen I, Kaur P, Nandicoori VK, Khosla S. Mycobacteria modulate host epigenetic machinery by Rv1988 methylation of a non-tail arginine of histone H3. Nat Commun 2015; 6:8922.

39. Chandran A, Antony $C$, Jose L, et al. Mycobacterium tuberculosis infection induces HDAC1-mediated suppression of IL-12B gene expression in macrophages. Front Cell Infect Microbiol 2015; 5:90.

40. Jose L, Ramachandran R, Bhagavat R, et al. Hypothetical protein Rv3423.1 of

- Mycobacterium tuberculosis is a histone acetyltransferase. FEBS J 2016; 283:265-281.

The study reports on the isolation and characterization of a Mycobacterial enzymeproducing euchromatic histone marks on histone $\mathrm{H} 3$.

41. Tailleux L, Neyrolles $O$, Honore-Bouakline $S$, et al. Constrained intracellular survival of Mycobacterium tuberculosis in human dendritic cells. J Immunol 2003; 170:1939-1948.

42. Pacis $A$, Tailleux $L$, Morin $A M$, et al. Bacterial infection remodels the DNA methylation landscape of human dendritic cells. Genome Res 2015; 25:1801-1811.

43. Esterhuyse MM, Weiner J, Caron $\mathrm{E}$, et al. Epigenetics and proteomics join transcriptomics in the quest for tuberculosis biomarkers. MBio 2015; 6:e01187-e01115

44. Richards AM, Abu Kwaik Y, Lamont RJ. Code blue: acinetobacter baumannii, a nosocomial pathogen with a role in the oral cavity. Mol Oral Microbiol 2015; 30:2-15.

45. Moon DC, Choi CH, Lee SM, et al. Nuclear translocation of acinetobacter baumannii transposase induces DNA methylation of $\mathrm{CpG}$ regions in the promoters of E-cadherin gene. PLoS One 2012; 7:e38974.

46. Ito N, Ohta K. Reprogramming of human somatic cells by bacteria. Dev Growth Differ 2015; 57:305-312.

47. Masaki T, Qu J, Cholewa-Waclaw J, et al. Reprogramming adult Schwann cells to stem cell-like cells by leprosy bacilli promotes dissemination of infection. Cell 2013; 152:51-67.

48. Hess S, Rambukkana A. Bacterial-induced cell reprogramming to stem celllike cells: new premise in host-pathogen interactions. Curr Opin Microbiol 2015; 23:179-188.

49. Fallows D, Peixoto B, Kaplan G, Manca C. Mycobacterium leprae alters - classical activation of human monocytes in vitro. J Inflamm (Lond) 2016; 13:8. The study describes how the interaction with $M$. leprae or its PGL affects cytokine production by human monocytes.

50. Polycarpou A, Holland MJ, Karageorgiou I, et al. Mycobacterium leprae

- activates Toll-like receptor-4 signaling and expression on macrophages depending on previous Bacillus Calmette-Guerin vaccination. Front Cell Infect Microbiol 2016; 6:72.

The study describes that irradiated, killed M. leprae activated TLR4 in a reporter cell line and stimulated cytokine production by human macrophages.

51. Naqvi AA, Ahmad F, Hassan Ml. Identification of functional candidates amongst hypothetical proteins of mycobacterium leprae Br4923, a causative agent of leprosy. Genome 2015; 58:25-42.

52. Kilian M, Chapple IL, Hannig M, et al. The oral microbiome - an update for oral

nealthcare professionals. Br Dent J 2016; 221:657-666.

Excellent review on the biofoilm-forming oral bacterial community and its dysbiotic alterations in oral diseases.

53. Takahashi N. Oral microbiome metabolism: from 'who are they?' to 'what are they doing?'. J Dent Res 2015; 94:1628-1637.

54. Hettmann A, Demcsak A, Decsi $G$, et al. Infectious agents associated with

- head and neck carcinomas. Adv Exp Med Biol 2016; 897:63-80.

The study analyses the relative contribution of human oncoviruses, bacteria, and fungi to the initiation and progression of malignant tumours in the head and neck region. 
55. Perera M, Al-Hebshi NN, Speicher DJ, et al. Emerging role of bacteria in oral - carcinogenesis: a review with special reference to perio-pathogenic bacteria. J Oral Microbiol 2016; 8:32762.

The review evaluates the reports on the association of distinct oral bacteria with oral carcinoma and discusses the potential pathogenic mechanisms involved.

56. Flynn KJ, Baxter NT, Schloss PD. Metabolic and community synergy of oral - bacteria in colorectal cancer. mSphere 2016; 1 :.

The review evaluates the reports on the association of distinct oral bacteria with colon carcinoma and proposes a polymicrobial model of colorectal carcinogenesis.

57. How KY, Song KP, Chan KG. Porphyromonas gingivalis: an overview of - periodontopathic pathogen below the gum line. Front Microbiol 2016; 7:53. The review deals with the virulence factors of the 'keystone pathogen' $P$. gingivalis.

58. Olsen I, Hajishengallis G. Major neutrophil functions subverted by Porphyr- omonas gingivalis. J Oral Microbiol 2016; 8:30936.

The review discusses how $P$. gingivalis impairs polymorphonuclear leukocytes and promotes the inflammatory changes characteristic for periodontitis.

59. Hajishengallis G, Darveau RP, Curtis MA. The keystone-pathogen hypothesis. Nat Rev Microbiol 2012; 10:717-725.

60. Barros SP, Williams R, Offenbacher S, Morelli T. Gingival crevicular fluid as a - source of biomarkers for periodontitis. Periodontol 2000 2016; 70:53-64. The review deals with disease markers which accumulate in abnormally deepened gingival sulcuses during periodontitis.

61. Frias-Lopez J, Duran-Pinedo A. Effect of periodontal pathogens on the metatranscriptome of a healthy multispecies biofilm model. J Bacteriol 2012; 194:2082-2095.

62. Barbosa GM, Colombo AV, Rodrigues PH, Simionato MR. Intraspecies variability affects heterotypic biofilms of Porphyromonas gingivalis and prevotella intermedia: evidences of strain-dependence biofilm modulation by physical contact and by released soluble factors. PLoS One 2015; 10:e0138687.

63. Gui MJ, Dashper SG, Slakeski N, et al. Spheres of influence: Porphyromonas gingivalis outer membrane vesicles. Mol Oral Microbiol 2016; 31:365-378.

The review focuses on the role of OMV in disease development and nutrition capture by $P$. gingivalis.

64. Polak D, Ferdman O, Houri-Haddad Y. Porphyromonas gingivalis capsule-

- mediated coaggregation as a virulence factor in mixed infection with fusobacterium nucleatum. J Periodontol 2016; 1-15.

The study describes an increased coaggregation of capsulated versus noncapsulated $P$. gingivalis with Fusobacterium nucleatum resulting in the aggravation of periodontitis in an experimental mouse model.

65. El-Awady AR, Miles $B$, Scisci $E$, et al. Porphyromonas gingivalis evasion of autophagy and intracellular killing by human myeloid dendritic cells involves DC-SIGN-TLR2 crosstalk. PLoS Pathog 2015; 10:e1004647.

66. Takeuchi $\mathrm{H}$, Takada $\mathrm{A}$, Kuboniwa $\mathrm{M}$, Amano $\mathrm{A}$. Intracellular periodontal - pathogen exploits recycling pathway to exit from infected cells. Cell Microbiol 2016; 18:928-948.

The study describes how vesicle-associated membrane protein and other regulators of membrane trafficking are recruited to early endosomes by $P$. gingivalis.
67. Benakanakere $M$, Abdolhosseini $M$, Hosur K, et al. TLR2 promoter hypermethylation creates innate immune dysbiosis. J Dent Res 2015; 94: 183-191.

68. Martins MD, Jiao $\mathrm{Y}$, Larsson $\mathrm{L}$, et al. Epigenetic modifications of histones in periodontal disease. J Dent Res 2016; 95:215-222.

69. Aruni AW, Zhang K, Dou Y, Fletcher H. Proteome analysis of coinfection of epithelial cells with filifactor alocis and Porphyromonas gingivalis shows modulation of pathogen and host regulatory pathways. Infect Immun 2014; 82:3261-3274.

70. Aruni AW, Mishra A, Dou Y, et al. Filifactor alocis: a new emerging periodontal pathogen. Microbes Infect 2015; 17:517-530.

71. Barros SP, Offenbacher S. Modifiable risk factors in periodontal disease: epigenetic regulation of gene expression in the inflammatory response. Periodontol 2000 2014; 64:95-110.

72. Ye F, Karn J. Bacterial short chain fatty acids push all the buttons needed to reactivate latent viruses. Stem Cell Epigenet 2015; 2:e532.

73. Kato $\mathrm{A}$, Imai $\mathrm{K}$, Ochiai $\mathrm{K}$, et al. Prevalence and quantitative analysis of Epstein-Barr virus DNA and Porphyromonas gingivalis associated with Japanese chronic periodontitis patients. Clin Oral Investig 2015; 19: 1605-1610.

74. $\mathrm{Lu} \mathrm{H}$, Zhu C, Li F, et al. Putative periodontopathic bacteria and herpesviruses in pregnant women: a case-control study. Sci Rep 2016; 6:27796.

75. Prusty BK, Gulve N, Rasa S, et al. Possible chromosomal and germline

- integration of human herpesvirus 7 (HHV-7). J Gen Virol 2016.

The study demonstrates integration of an HHV7 genome into the human germline.

76. Kuhl U, Lassner D, Wallaschek N, et al. Chromosomally integrated human herpesvirus 6 in heart failure: prevalence and treatment. Eur J Heart Fail 2015; 17:9-19.

77. Agut $H$, Bonnafous $P$, Gautheret-Dejean $A$. Update on infections with human herpesviruses 6A, 6B, and 7. Med Mal Infect 2016

78. Subbarayal $P$, Karunakaran $K$, Winkler $A C$, et al. EphrinA2 receptor (EphA2) is an invasion and intracellular signaling receptor for Chlamydia trachomatis. PLoS Pathog 2015; 11:e1004846.

79. Gupta G, Song J. C-terminal auto-regulatory motif of hepatitis C virus NS5B interacts with human VAPB-MSP to form a dynamic replication complex. PLoS One 2016; 11:e0147278.

80. Campadelli-Fiume G, Collins-McMillen D, Gianni T, Yurochko AD. Integrins as herpesvirus receptors and mediators of the host signalosome. Annu Rev Virol 2016; 3:215-236.

81. Rolando M, Gomez-Valero L, Buchrieser C. Bacterial remodelling of the host epigenome: functional role and evolution of effectors methylating host histones. Cell Microbiol 2015; 17:1098-1107.

82. Chumduri C, Gurumurthy RK, Zietlow R, Meyer TF. Subversion of host genome integrity by bacterial pathogens. Nat Rev Mol Cell Biol 2016; 17:659-673.

83. Prusty BK, Krohne G, Rudel T. Reactivation of chromosomally integrated human herpesvirus- 6 by telomeric circle formation. PLoS Genet 2013; 9:e1004033. 